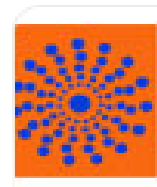

JOURNAL OF BEHAVIOR, HEALTH \& SOCIAL ISSUI

inis
Journal of Behavior, Health \& Social Issues ISSN: 2007-0780

jcpedro@unam.mx

Asociación Mexicana de Comportamiento y Salud, A. C.

México

Patrón-Espinosa, Felipe de Jesús

LA EVITACIÓN EXPERIENCIAL COMO DIMENSIÓN FUNCIONAL DE LOS TRASTORNOS DE DEPRESIÓN, ANSIEDAD Y PSICÓTICOS

Journal of Behavior, Health \& Social Issues, vol. 5, núm. 1, mayo-octubre, 2013, pp. 85-95

Asociación Mexicana de Comportamiento y Salud, A. C.

Distrito Federal, México

Disponible en: http://www.redalyc.org/articulo.oa?id=282227878007

Cómo citar el artículo

- Número completo

- Más información del artículo

- Página de la revista en redalyc.org

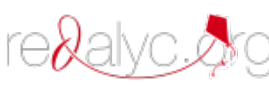

Sistema de Información Científica

Red de Revistas Científicas de América Latina, el Caribe, España y Portugal Proyecto académico sin fines de lucro, desarrollado bajo la iniciativa de acceso abierto 


\title{
LA EVITACIÓN EXPERIENCIAL COMO DIMENSIÓN FUNCIONAL DE LOS TRASTORNOS DE DEPRESIÓN, ANSIEDAD Y PSICÓTICOS
}

\author{
THE EXPERIENTIAL AVOIDANCE AS FUNCTIONAL DIMENSIONS OF \\ DEPRESSION, ANXIETY AND PSYCHOTIC DISORDERS
}

Felipe de Jesús Patrón-Espinosa

Universidad Autónoma de Yucatán, Mérida, México.

\author{
Recibido: Marzo 20, 2013 \\ Revisado: Abril 09, 2013 \\ Aprobado:Abril 28, 2013 \\ Línea de investigación: 1. Psicología clínica levaluación, \\ intervenciones basada en evidencial. 2. Psicología básica; \\ investigación de laboratorio que explora cómo el variar las \\ propiedades espaciales de los eventos de estímulo influye en el \\ aprendizaje de sujetos no humanos. \\ Dirección postal: Universidad Autónoma de Yucatán, Facultad \\ de Psicología, Calle 31-A Número 300 Fraccionamiento San \\ Esteban. Mérida, Yucatán, México, CP: 97149 \\ Correo: patron6d5ahotmail.com
}

Resumen

Recientemente la Evitación Experiencial (Hayes, Wilson, Gifford, Follette, \& Strosahl, 1996) se ha propuesto como un elemento compartido por distintos síndromes psiquiátricos. Diversos estudios, realizados fuera de México en su mayoría, han contado con el objetivo de identificar si existe alguna relación entre la Evitación Experiencial (EE) y otros trastornos psiquiátricos. Siguiendo esta línea, el presente estudio contó con el propósito de identificar diferencias en los niveles de Evitación Experiencial entre personas con un diagnóstico psiquiátrico (trastorno de ansiedad, depresión y psicótico) y población abierta de la ciudad de Mérida Yucatán. Se aplicó el Acceptance and Action Questionnaire II adaptado para población yucateca (Patrón, 2010) a 108 participantes. La muestra se dividió en cuatro grupos de 27 integrantes dependiendo del diagnóstico: (1) ansiedad, (2) depresión, (3) psicótico y (4) no clínico. Se realizó un análisis estadístico aplicando una prueba de varianza simple. Los resultados sugieren que existen diferencias estadísticamente significativas entre el grupo no clínico y los grupos clínicos, así como entre el grupo con diagnóstico de trastorno psicótico y los grupos con trastorno de depresión y ansiedad. Estos hallazgos promueven estudiar con mayor profundidad el papel que juega la EE en los distintos tipos de esquizofrenia, así como a proponer posibles técnicas dirigidas a influir sobre la EE como parte del tratamiento de diversos trastornos. Palabras clave: Evitación Experiencial, Trastornos Psiquiátricos, AAQ-II. 
Abstract

Lately the experiential avoidance (Hayes, Wilson, Gifford, Follette, \& Strosahl, 1996) has been proposed as an element shared by different psychiatric syndromes. Several studies have been conducted outside of Mexico with the aim to identify whether there is any relationship between experiential avoidance (EA) and other psychiatric disorders. Following this argument, the present study was conducted to identify differences in the levels of experiential avoidance among people who have a psychiatric diagnosis (anxiety, depression and psychotic disorder) and non-clinical population from the city of Mérida Yucatán. The Acceptance and Action Questionnaire II adapted for Yucatán population (Patrón, 2010) was applied to 108 participants. The sample was divided into four groups of 27 members depending on the diagnosis: (1) anxiety, (2) depression, (3) psychotic and (4) non-clinical. Statistical analysis was performed using simple variance test. The results suggest that there were significant differences between clinical and nonclinical groups and between the group with a diagnosis of psychotic disorder and groups with depression and anxiety disorder. These findings lead to study in more depth the role that the EA plays in the different types of schizophrenia, and to suggest possible techniques designed to influence the EA as part of the treatment of various disorders.

Key words: Experiential Avoidance, Psychiatric Disorders, AAQ-II.

\section{Introducción}

Una de las aportaciones más recientes dentro del Análisis Experimental de la Conducta al abordaje conceptual de la cognición y el lenguaje es la Teoría de los Marcos relacionales (Hayes, 1991, 1994; Hayes, Barnes-Holmes \& Roche, 2001). El fundamento teórico y empírico de esta propuesta se origina con los estudios realizados por Sidman (1971), en los que propone el concepto de equivalencia de estímulos. En éstos, Sidman describe el caso de un sujeto microcefálico con problemas de aprendizaje que había logrado igualar palabras habladas (A) con imágenes (B). Con este entrenamiento se consiguió que el sujeto igualara imágenes (B), como muestras, a palabras escritas (C) como comparaciones, y viceversa (relación C-B). También se consiguió que el paciente nombrara las palabras escritas (relación C-A). Tales relaciones "no entrenadas" generalmente se denominan como equivalencia de estímulos.

A partir de este descubrimiento inicia una línea de investigación que termina identificando tres propiedades básicas con respecto a las relaciones de equivalencia: la reflexividad, la simetría y la transitividad (García, 2002). Continuando con la simbolización empleada en el ejemplo anterior, es posible definir dichas propiedades como sigue:

1. Reflexividad: Intercambiabilidad de un elemento consigo mismo $(A=A)$.
2. Simetría: Inversión de la relación muestraestímulo de comparación ( $\mathrm{Si} A=\mathrm{B}$, entonces $\mathrm{B}=\mathrm{A})$.

3. Transitividad: Transferencia entre dos discriminaciones condicionales mediada por algún elemento compartido ( $\mathrm{Si} \mathrm{A}=\mathrm{B}$ y $\mathrm{B}=\mathrm{C}$, entonces, $\mathrm{A}=\mathrm{C}$ ).

Dentro de la postura de la Teoría de los Marcos Relacionales (TMR), se toma a la equivalencia de estímulos como una de las muchas clases de respuestas (Skinner, 1935) definidas por la actividad de enmarcar eventos relacionalmente. Este tipo de respuestas son el punto central de la TMR, y se caracterizan por la sustitución de funciones pertinentes a un estímulo hacia otro estímulo nuevo (Hayes, 1991). De esta manera, las respuestas relacionales son concebidas como una operante generalizada, es decir, como un conjunto de respuestas definidas funcionalmente donde los miembros de una clase difieren en sus características topográficas, pero comparten la misma contingencia que determina su efecto sobre el entorno. Así, este tipo de respuestas pueden formar clases operantes generalizadas a partir de una historia de entrenamiento similar (Wilson \& Luciano, 2002).

Partiendo de lo anterior es posible definir un marco relacional como un patrón particular de respuesta controlada contextual y arbitrariamente 
que se estructura a partir de una historia de reforzamiento diferencial ante claves contextuales. Cuando un organismo responde de esta forma no lo hace al contenido físico de los estímulos, sino al marco relacional, es decir a las claves contextuales establecidas históricamente, de manera que cuando el organismo responde su respuesta es enmarcar los eventos relacionalmente (Hayes et al., 1999).

La conducta verbal, así como la simbólica, desde esta perspectiva son el mismo fenómeno observado por Sidman (1971) con la equivalencia de estímulos, pues un estímulo es verbal si parte de sus funciones vienen dadas por su participación en marcos relacionales. De esta manera, cualquier estímulo que sea verbal dependiendo de su historial de reforzamiento y su contexto puede producir conducta derivada, transferencia o transformación de funciones por su relación con otros estímulos (Gómez-Martín, López-Ríos, \& Mesa-Majón, 2007).

A pesar de que la capacidad humana para derivar y producir transferencia o transformación de funciones tiene grandes ventajas adaptativas, también posee ciertas características que favorecen el desarrollo de problemas psicológicos. En general, estas características se pueden considerar como parte del lenguaje dentro del cual se identifican cuatro causas que multiplican el sufrimiento humano: (1) la literalidad o bidireccionalidad que es un producto ineludible del comportamiento verbal e implica responder a un evento en términos de otro en virtud de las propiedades del repertorio relacional (los vínculos mutuos y combinatorios y la transformación de funciones). (2) La formación de categorías o evaluación es la tendencia a evaluar casi todo debido a la literalidad y a no distinguir entre las propiedades intrínsecas de un evento ("estoy triste") y sus propiedades arbitrarias establecidas socialmente ("estar triste es malo"). (3) El dar razones y la búsqueda de coherencia viene potenciado por el punto de vista cultural de que el comportamiento se explica (erróneamente) por las emociones y los pensamientos (por ejemplo, "estos pensamientos son terribles y no puedo vivir con ellos, no puedo trabajar, no puedo estar con mis hijos, etc."). (4) La búsqueda de control de los eventos privados es el contexto clave que da sentido a los anteriores en tanto que es actuar siguiendo esas razones paradójicas, por ejemplo, comportándose fusionado a pensamientos molestos tomados como causas (Luciano \& Sonsoles, 2006).

De esta manera, el contexto nos enseña por medio del lenguaje a clasificar ciertos eventos privados como "malos" al asociarlos con determinadas sensaciones corporales, predisposiciones conductuales, etc. Como el lenguaje (pensamiento) representa a estos estados aversivos, por ejemplo: "tengo miedo de que me ponga nervioso cuando declame", éstos por sí solos pueden invocar los síntomas sin necesidad de la situación real. Por lo tanto, el intento de escapar o evitar de tal situación no es suficiente para liberarse del estado aversivo.

Este patrón de comportamiento de escape o evitación es conocido como Trastorno de Evitación Experiencial (TEE). Hayes et al. (1996), lo definen como "un fenómeno que ocurre cuando una persona no está dispuesta a ponerse en contacto con experiencias privadas particulares (v.g: sensaciones corporales, emociones, pensamientos, recuerdos, predisposiciones conductuales) e intentar alterar la forma o la frecuencia de esos eventos y el contexto que los ocasiona" (p. 1156).

Según Wilson y Luciano (2002), una persona atrapada en este patrón recurrente de evitación estaría inmersa en un círculo vicioso en el que ante la presencia de cualquier función verbal aversiva, se produce la necesidad de aplacarla para lo que hará lo que considere correcto de acuerdo con su historia. Contradictoriamente, aunque este patrón comportamental es aparentemente efectivo a corto plazo (en la medida en que consigue reducir o eliminar temporalmente una experiencia que genera malestar), si se convierte en crónico llega a producir una limitación en la vida de la persona. De acuerdo con Luciano y Sonsoles (2006), el problema es que tales actuaciones producen un alivio inmediato en ocasiones, pero provocan un efecto boomerang (es decir, el malestar vuelve a estar presente, a veces más intenso y extendido, y el alivio es breve). Esto obliga a no parar en el intento por hacer desaparecer el malestar, que a su vez, estará cada vez más presente por el efecto boomerang. 
A partir de la delimitación del TEE se le ha propuesto como un elemento compartido por una buena parte de los síndromes que conforman los sistemas de clasificación sindrómicos tradicionales como el Manual diagnóstico y estadístico de trastornos mentales (DSM) y la Clasificación internación de enfermedades (CIE) (Hayes et al., 1996). Esta afirmación se justifica argumentando que las distintas etiquetas diagnósticas se estipulan a partir de diferentes contenidos "cognitivos" o reacciones ante distintos estímulos o circunstancias, pero quitando la atención de las propiedades topográficas de las respuestas o los estímulos, existe en la gran mayoría de estos síndromes un elemento común, la evitación experiencial. Con el fin de clarificar esta propuesta se presenta un ejemplo hipotético de la vida común. En el caso de una persona que consume alguna sustancia psicoactiva de manera crónica, el interés desde una perspectiva psicológica no es si consume heroína, cocaína o alcohol, sino el patrón de comportamiento que mantiene dichos niveles de consumo y que posiblemente afectan otras áreas importantes o relevantes de la vida del individuo. Este patrón de comportamiento puede ser descrito como evitación experiencial si el consumo de dicha sustancia alivia a la persona al "hacerlo olvidar" o "reducir las penas" que constantemente lo persiguen. Como sería evidente, el patrón de consumo está siendo mantenido por la evitación de eventos privados que son aversivos para la persona.

Siguiendo con esta idea, Luciano y Sonsoles (2006) mencionan que la evitación experiencial se concibe, desde la TMR, como una dimensión funcional que sirve de base a numerosos trastornos y es un modo radicalmente diferente de presentar y entender los desórdenes psicológicos o mentales; entendiendo la psicopatología desde esta perspectiva genuinamente psicológica, muy distante de las aproximaciones reduccionistas, en particular, las biologicistas.

En la actualidad, existen diversos estudios que en cierta medida confirman la hipótesis anterior. En éstos se ha encontrado relación entre el TEE y otros desordenes psicológicos, por ejemplo, trastornos de ansiedad y psicóticos (Vargas \& Aguilar, 2006), psicosomáticos (Rodríguez, 2002), de adicciones (Velasco \& Quiroga, 2001), sinto- matología delirante (García, Luciano, Hernández, \& Zaldivar, 2004), síntomas psicóticos (GarcíaMontes, Pérez-Álvarez, \& Cangas-Díaz, 2006), entre otros. Para una revisión más detallada consultar en Wilson y Luciano (2002).

Partiendo de la hipótesis anteriormente planteada, Vargas y Aguilar (2006) realizaron el primer estudio en el que se evaluó el TEE en México. Su propósito fue medir los niveles de Evitación Experiencial (EE) en personas que contaban con un diagnóstico psiquiátrico, ya sea psicótico o neurótico, así como en población abierta. La muestra se conformó por cinco pacientes psicóticos (esquizofrenia), cinco pacientes neuróticos (trastornos de ansiedad), y cinco participantes no clínicos. El instrumento que emplearon los autores para dicho propósito fue una versión adaptada para México del AAQ-I (Hayes et al., 2004). Anteriormente, Barraca (2004) ya había traducido una versión al castellano para población española. La primera versión en inglés mostró un índice de consistencia interna (alfa de Cronbach) de 0.70 , mientras que la versión realizada por Barraca mostró un índice de 0.74.

Estas tres versiones del AAQ-I, incluyendo la adaptación realizada por Vargas y Aguilar (2006), se caracterizan por ser una escala tipo Likert que consta de 9 reactivos con 7 opciones de respuesta de que van de nunca (never) a siempre (always). Los resultados obtenidos por Vargas y Aguilar (2006) demostraron que los niveles de EE fueron menores en los sujetos no clínicos en comparación con los pacientes que contaban con un diagnóstico psiquiátrico. De igual manera, se registró un menor nivel de EE en los pacientes neuróticos en comparación con los psicóticos.

A pesar de que los resultados obtenidos por Vargas y Aguilar (2006) corroboran lo reportado por los estudios mencionados anteriormente, en el sentido de una relación entre la EE y diferentes trastornos psiquiátricos, existe una característica que impide su generalización. En específico, el instrumento empleado por Vargas y Aguilar no pasó por un proceso de estandarización.

Realizar un estudio que apoye los resultados obtenidos por Vargas y Aguilar empleando una escala estandarizada es de suma importancia debido a que en México no se ha evaluado anteriormente el TEE. Para dicha tarea existe el 
Acceptance and Action Questionnaire II (AAQ-II), que es una escala que ha sido estandarizada para población yucateca y su objetivo es identificar el nivel de EE en los participantes (Patrón, 2010). Éste es una adaptación del AAQ-II creado por Bond et al. (2011) que en su versión en inglés mostró un índice de consistencia interna de 0.84, así como valores de EE más altos en personas con niveles elevados de depresión, ansiedad, estrés y angustia psicológica. En relación al AAQ-II para población yucateca, sus características psicométricas se describen a detalle más adelante en el apartado de instrumentos, por el momento, es relevante resaltar que de encontrar resultados que coincidan con los hallazgos de Vargas y Aguilar (2006), se apoyaría la hipótesis que concibe al TEE como una dimensión funcional en distintos desórdenes psicológicos, y le brindaría mayor generalización al confirmarse con población mexicana. Esto podría aportar una comprensión de los desórdenes psiquiátricos distinta a la que se ha empleado mayormente hasta ahora, así como fomentar la investigación y desarrollo de intervenciones que aborden el tratamiento del TEE como terapia alternativa o complementaria a los trastornos estudiados.

Bajo esta lógica, y con el propósito de buscar evidencia que apoye los resultados obtenidos por Vargas y Aguilar (2006), el objetivo de este trabajo es identificar si existen diferencias significativas en los niveles de EE entre personas con un diagnóstico psiquiátrico (trastorno de ansiedad, depresión y psicótico) y personas sin diagnóstico clínico de la ciudad de Mérida Yucatán. Esto empleando una escala estandarizada (AAQ-II para población yucateca).

Los diversos diagnósticos empleados para conformar los grupos se eligieron continuando con el tipo de selección realizada por Vargas y Aguilar (2006), sin embargo, a diferencia de este estudio, en el presente el análisis se extiende a desordenes depresivos debido a las siguientes razones: (1) la depresión es el segundo trastorno psiquiátrico con mayor prevalencia en México, solamente superado por la ansiedad (Wagner, González-Forteza, Sánchez-García, García-Peña \& Gallo, 2012). Consideramos imperante abordar dicho trastorno por su afectación en la salud pública. (2) Debido a que el TEE se plantea como dimensión funcional presente en diversos trastornos psiquiátricos, independientemente de la topografía de respuesta que los caracterice, consideramos pertinente extender el análisis a otros desórdenes con el fin de obtener mayor generalización en la comprobación de la hipótesis.

\section{Método}

\section{Participantes}

El tipo de muestreo fue por disponibilidad (no aleatorio) y participaron 108 personas residentes de la ciudad de Mérida, Yucatán, de las cuales la edad promedio fue de 35.8 años (d.e.=12.2), con un valor mínimo de 18 y máximo de 61 años. El 58\% de los participantes se identificó con el género femenino. La muestra constó de cuatro grupos que se conformaron por participantes que sabían leer y escribir. El primer grupo consistió de 27 participantes no clínicos, los tres grupos restantes contaron con un solo diagnóstico basado en las categorías estipuladas en el CIE-10, es decir, que las personas que presentaron comorbilidad no fueron seleccionados. Así, 27 participantes contaban con un diagnóstico de trastorno de ansiedad (reacción al estrés grave y trastornos de adaptación), 27 con un diagnóstico de trastorno psicótico (esquizofrenia paranoide) y 27 con un diagnóstico de trastorno depresivo (depresivo recurrente) (ver Tabla 1).

Los participantes clínicos se encontraban en consulta externa al momento de ser abordados, a excepción de los pacientes psicóticos, los cuales se encontraban institucionalizados. Los pacientes clínicos se obtuvieron del Hospital Psiquiátrico "Yucatán", excepto la mitad de los que contaban con diagnóstico de trastorno psicótico, quienes se encontraban institucionalizados en el grupo "Amanecer nuevamente" de la ciudad de Mérida Yucatán. La estrategia de tomar participantes de una organización diferente fue necesaria debido a la poca población de pacientes con un diagnóstico de trastorno psicótico en el Hospital Psiquiátrico "Yucatán". El 50\% de los participantes no clínicos fue tomando de la licenciatura en Informática de un centro universitario de la ciudad de Mérida. Para el $50 \%$ restante se seleccionaron empleados sin estudios universitarios del área comercial y de servicios de la ciudad de Mérida. 
Tabla 1.

Análisis de frecuencias de la variable género y media y desviación estándar de la edad de los participantes.

\begin{tabular}{|c|c|c|c|c|}
\hline Grupos & Masculino & Femenino & Media de la edad & d. e. edad \\
\hline No clínico & 14 & 13 & 30.4 & 11.8 \\
\hline T. Depresivo & 7 & 20 & 40.7 & 12.7 \\
\hline T. Ansiedad & 9 & 18 & 36.1 & 10.1 \\
\hline T. Psicótico & 15 & 12 & 36 & 12.4 \\
\hline Total & 45 & 63 & 35.8 & 12.2 \\
\hline
\end{tabular}

Instrumentos

Acceptance and Action Questionnaire-II (AAQII). Esta escala evalúa el nivel de EE y cuenta con un coeficiente de consistencia interna (alfa de Cronbach) $=.89$ para población Yucateca. Con respecto a su validez de constructo un análisis exploratorio de componentes principales con rotación varimax, estimó que la estructura de la escala es unifactorial, siendo la EE el factor que la compone. A partir de la aplicación el índice de correlación de Pearson entre los resultados del AAQ-II y el Inventario de Ansiedad de Beck (Tafoya-Ramos, PérezMitre, Ortega-Soto, \& Ortiz-León, 2006) se demostró su validez concurrente, al obtener una correlación positiva moderada (0.68). Para un análisis más detallado de las propiedades psicométricas del AAQ-II para población yucateca revisar la estandarización realizada por Patrón (2010). En relación al contenido, se caracteriza por ser una escala tipo Likert autoaplicada compuesta por 10 reactivos que describen diversas afirmaciones con respecto a cómo el individuo reacciona o se comporta ante eventos privados indeseables y qué tanto intenta controlarlos. Cada reactivo se puntúa de 1 a 7 , correspondiendo la puntuación 1 a "completamente falso" y la puntuación 7 a "completamente cierto". La puntuación total es la suma de las puntuaciones de todos los reactivos, empero los número 1,6 y 10 se suman de forma inversa. La mayor puntuación posible es 70 y ésta indicaría el grado más elevado de $\mathrm{EE}$, mientras que la menor puntuación posible es 10 .

\section{Procedimiento}

El primer paso para llevar a cabo la investigación fue solicitar la autorización a los directivos del Hospital Psiquiatrico "Yucatán" y del Grupo Amanecer Nuevamente, esto por medio de la Universidad Autónoma de Yucatán. Habiendo obtenido la autorización de ambas instituciones el aplicador del instrumento, que en este caso fue el autor del presente estudio, recibió entrenamiento para abordar a los participantes de acuerdo con los criterios éticos de cada institución. Este entrenamiento fue brindado por parte del responsable del área de investigación de dichas instituciones y constó del reconocimiento de las instalaciones, indicación del lugar y la forma en la que se abordaría a los participantes, como por ejemplo el saludo, la solicitud de participación, la firma del consentimiento informado, etc. En el Hospital 
Psiquiátrico "Yucatán" se inició la aplicación en consulta externa cuando los pacientes salían de la misma, el diagnóstico se obtuvo anteriormente por parte del psiquiatra en turno. Después se prosiguió a aplicar en el área de hospitalización para los participantes con trastornos psicóticos. Debido a la situación de institucionalización, la aplicación a estos participantes fue supervisada y apoyada por personal hospitalario. El procedimiento para abordar a los participantes del Grupo Amanecer Nuevamente fue similar, primero el aplicador obtuvo entrenamiento por parte del personal con respecto al abordaje de los pacientes, después se obtuvo el diagnóstico por parte del psiquiatra y finalmente se aplicó la escala, siempre con apoyo del personal hospitalario.

Cabe señalar que el diagnóstico fue llevado a cabo por los psiquiatras de las instituciones respectivas a partir del cumplimiento de los criterios de la CIE-10 y de las historias clínicas de los pacientes. Los participantes hospitalizados fueron seleccionados por el equipo hospitalario de ambas instituciones, con el fin de que éstos se encontraran en condiciones físicas apropiadas para entender y responder la escala, tomando en cuenta el nivel del trastorno padecido y los posibles efectos del tratamiento médico.

Los participantes no clínicos que contaban con estudios universitarios fueron obtenidos de un centro universitario de la ciudad de Mérida, en particular, eran estudiantes de la licenciatura en informática. La otra mitad, sin estudios universitarios, fueron seleccionados con el fin de no crear un sesgo por el nivel de educación al comparar el grupo no clínico con los grupos con diagnóstico.
De manera general, antes de iniciar la aplicación de la escala, el aplicador leyó y le permitió leer a todos los participantes una carta de consentimiento informado, en la que se mencionaba el nombre de la investigación, su objetivo, el responsable de la misma, así como todos los derechos con los que cuentan los participantes. Al terminar la lectura, y en caso de que la persona accediera a responder, se le solicitó firmara el consentimiento informado. Habiendo firmado, se procedía a la aplicación. Posteriormente, los datos fueron codificados manualmente. El análisis de datos se realizó por medio del software PASW Statistics $17.0^{\circledR} \mathrm{y}$ las gráficas por medio del software Sigmaplot $11.0^{\circledR}$ para ambiente Windows.

\section{Resultados}

Se empleó un análisis de varianza simple con el fin de determinar si existían diferencias en las medias del nivel de EE entre los grupos. Como se puede observar en la Tabla 2 y en la Figura 1, el análisis reveló que sí existen diferencias estadísticamente significativas entre los grupos $(F(3,104)=30.2 ; p=.0005, \eta 2=.47)$. La prueba post hoc de Scheffe $(\alpha=.05)$ reportó que existen diferencias estadísticamente significativas entre el grupo no clínico $(m=21.3$, d.e.=8.4) y todos los demás grupos clínicos, de los cuales el que contaba con diagnóstico de trastorno psicótico presentó un nivel de $\mathrm{EE}(\mathrm{m}=38.3$, d.e.=11.32) menor que el de los grupos con un trastorno de ansiedad $(\mathrm{m}=47.5$, d.e. $=14.5)$ o de depresión $(m=47.6$, d.e. $=11.8)$. Entre estos dos últimos el nivel de EE no mostró diferencias estadísticamente significativas.

Tabla 2.

Análisis de la varianza por diagnóstico para el grado de Evitación Experiencial.

\begin{tabular}{cccccccc}
\hline & No clínico & Depresivo & Ansiedad & Psicótico & $\mathrm{F}$ & $\mathrm{p}$ & $\eta 2$ \\
\hline EE & 21.3 & 47.6 & 47.5 & 38.3 & 30.2 & $.0005^{* * *}$ & .47 \\
\hline
\end{tabular}




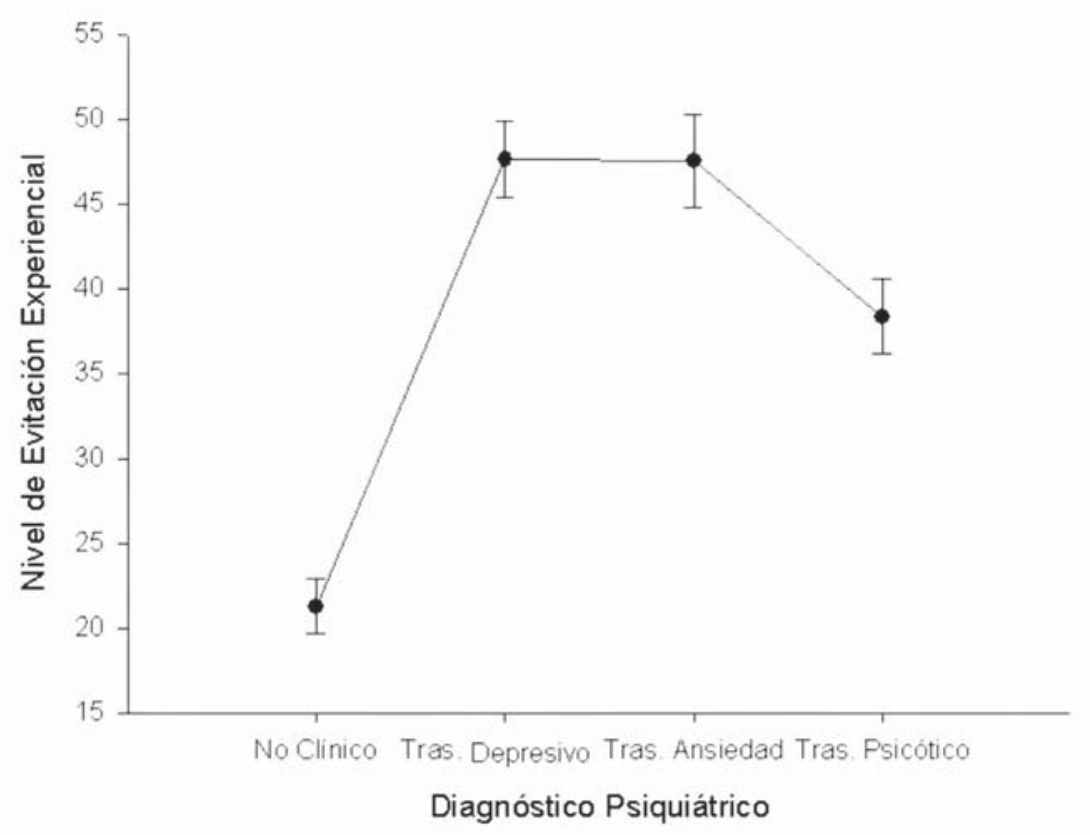

Figura 1. Comparación de las medias del nivel de Evitación Experiencial entre grupos según su diagnóstico, expresando el error simple.

\section{Discusión}

Consideramos que los resultados obtenidos no son concluyentes, pues a pesar de que de forma general los grupos con algún diagnóstico psiquiátrico cuentan con niveles mayores de EE en comparación con el grupo no clínico, las diferencias entre los grupos psiquiátricos no son claras. En primer lugar, los resultados revelan que los grupos con diagnóstico de trastorno depresivo y trastorno de ansiedad obtienen puntajes similares. Este resultado, aunque sea inesperado, es congruente con la propuesta teórica si se considera que la EE es un patrón de comportamiento de evitación ante eventos privados indeseables sin importar su categoría "emocional", por ejemplo tristeza o ansiedad; en otras palabras, la topografía de la respuesta no es el punto central. Siguiendo esta idea, podría aceptarse que los resultados no demuestren una diferencia en el nivel de EE entre los grupos con diagnóstico de ansiedad y depresión, pues la prueba AAQ-II reporta cierto grado de EE en ambos grupos pero no logra diferenciarlos, posiblemente porque su objetivo no es discriminar entre diferentes trastornos psiquiátricos.

Por otra parte, el resultado que muestra que el grupo con diagnóstico de trastorno psicótico cuenta con un menor nivel de EE en comparación con los grupos con diagnóstico de trastorno depresivo y de ansiedad es contradictorio a los resultados obtenidos por Vargas y Aguilar (2006), pues se esperaba un efecto inverso.

Sólo con fines especulativos, es posible proponer dos posibles explicaciones para este resultado. La primera se fundamenta en algunas afirmaciones que han realizado Bach y Hayes (2002) al exponer sobre los trastornos psicóticos y los delirios. Estos autores afirman que dichos trastornos pueden constituir una defensa en contra de los sentimientos de desesperanza o baja autoestima, lo cual podría suponer que los sujetos niegan sus síntomas. Concordando con esto, García et al. (2004) mencionan que la función de la EE en un trastorno neurótico es 
evitar el síntoma (ansiedad, obsesiones, tristeza, etc.), pero en el caso de un trastorno psicótico su función es más complicada, puesto que en éste, el síntoma vendría a ser él mismo una forma de evitación de algún otro problema. En síntesis, una posible explicación para las diferencias en el puntaje entre el grupo con diagnóstico de trastorno psicótico y los grupos con diagnóstico de trastorno de ansiedad y depresión, es que los participantes que integraban el grupo psicótico negaban sus síntomas. A pesar de que no consideramos pertinente afirmar que lo anterior explica los resultados inesperados, sí creemos que estas propuestas brindan un camino para posibles investigaciones a futuro que lidien con este problema.

La segunda explicación para este resultado inesperado hace referencia a limitaciones en la aplicación de los instrumentos. Se considera que especialmente en la aplicación del instrumento a pacientes psicóticos las condiciones no fueron las más adecuadas debido a que éstos se encontraban hospitalizados, situación que incluye muchas variables extrañas imposibles de controlar al hacer investigación en el ámbito clínico, en especial cuando la labor del investigador queda subordinada a esferas más importantes como resguardar la integridad del participante, cumplir el protocolo ético de la institución y no afectar el tratamiento proporcionado por los profesionales especialistas. A esto habría que agregar otras posibles variables extrañas como la medicación de los participantes, o posibles diferencias en el abordaje a los participantes debido a las características físicas de las instituciones o al entrenamiento proporcionado al aplicador.

\section{Conclusiones}

A pesar de que en cierta medida los resultados obtenidos fueron contradictorios, se considera que este estudio cumple su objetivo y aporta evidencia a favor de la hipótesis según la cual el TEE es una dimensión funcional en distintos desórdenes psicológicos, pues el nivel de EE mostrado por el grupo no clínico fue menor al de los demás grupos. Además, con el empleo del
AAQ-II en este estudio, se confirma que es un instrumento que puede aplicarse a personas con un nivel de estudios básico, esta característica en combinación con su longitud (10 reactivos) lo hacen una herramienta apta para la investigación en el ámbito clínico.

Próximos estudios pueden estar dirigidos a explorar cuál es el papel que juega la EE en los trastornos psicóticos y si los resultados contradictorios obtenidos en este estudio fueron debidos a una función particular de la EE ante este tipo de desórdenes o por problemas metodológicos.

Finalmente, estos resultados dan una posible explicación del por qué intervenciones enfocadas al tratamiento del TEE, como la Terapia de Aceptación y Compromiso (Hayes, Strosahl, \& Wilson, 1999), muestran ser efectivas para distintos trastornos, por ejemplo, abuso de sustancias (Twohig, Shoenberger, \& Hayes, 2007), psicosis (Pankey \& Hayes, 2003), agorafobia (Carrascoso, 2000), estrés y dolor (Dahl, Wilson, \& Nilsson, 2004), o sintomatología delirante (García, Luciano, Hernández, \& Zaldivar, 2004), lo que podría sugerir posibles beneficios al tomar en cuenta este tipo de técnicas en el tratamiento de diversos desórdenes psiquiátricos.

\section{Referencias}

Bach, P. \& Hayes, S.C. (2002). The use of Acceptance and Commitment Therapy to prevent the rehospitalization of psychotic patients: A randomized controlled trial. Journal of Consulting and Clinical Psychology, 70, 11291139, available via: http://dx. doi:10.1037/0022006X.70.5.1129

Barraca, M. J. (2004). Spanish adaptation of Acceptance and Action Questionnaire (AAQ). International Journal of Psychology and Psychological Therapy, 4, 550-515.

Bond, F. W., Hayes, S. C., Baer, R. A., Carpenter, K. C., Guenole, N., Orcutt, H. K., et al. (2011). Preliminary psychometric properties of the Acceptance and Action Questionnaire - II: A revised measure of psychological flexibility and acceptance. Behavior Therapy, 42, 676688, available via: http://dx.doi:10.1016/j. beth.2011.03.007 
Carrascoso, F. J. (2000). Acceptance and Commitment Therapy (ACT) in panic disorder with agoraphobia: a case study. Psychology in Spain, 4 (1), 120-128.

Dahl, J., Wilson, K. \& Nilsson, A. (2004). Acceptance and Commitment Therapy and the treatment of persons at risk for long-term disability resulting from stress and pain symptoms: a preliminary randomized trial. Behavior Therapy 35, 785801.

García, A. (2002). Orígenes, ampliación y aplicaciones de la equivalencia de estímulos. Apuntes de psicología. 20(2), 171-186.

García, J., Luciano, C., Hernández, M., \& Zaldivar, F. (2004). Aplicación de la terapia de aceptación y Compromiso (ACT) a sintomatología delirante: un estudio de caso. Psicothema, 16, 117-124.

García-Montes, J., Pérez-Álvarez, M., \& CangasDíaz, A. (2006). Aproximación al abordaje clínico de los síntomas psicóticos desde la Aceptación. Apuntes de Psicología, 24(1-3), 293-307.

Gómez-Martín, S., López-Ríos, F., \& Mesa-Majón, H. (2007). Teoría de los Marcos Relacionales: algunas implicaciones para la psicopatología y la psicoterapia. International Journal of clinical and health psychology, 7, 491-507.

Hayes, S. C. (1991). A relational control theory of stimulus equivalence. En Hayes, L. J. \& Chase, P. N. (Eds.) Dialogues on verbal behavior (pp. 19-40). Reno, NV: Context Press.

Hayes, S. C. (1994). Relational frame theory: A functional approach to verbal events. En S. C. Hayes, L. J. Hayes, M. Sato, \& K. Ono, Behavior Analysis of language and cognition (pp. 9-30). Reno, NV: Context Press.

Hayes, S. C., Barnes-Holmes, D., \& Roche, B. (2001). Relational frame theory: a postskinnerian account of human language and cognition. New York: Plenum

Hayes, S. C., Bissett, R., Korn, Z., Zettle, R. D., Rosenfarb, I., Cooper, I., et al. (1999). The impact of acceptance versus control rationales on pain tolerance. The Psychological Record, 49, 33-47.

Hayes, S. C., Strosahl, K. D. \& Wilson. K. G. (1999). Acceptance and commitment therapy: An experiential approach to behavior change. New York, NY, US: The Guilford Press
Hayes, S. C., Strosahl, K. D., Wilson, K. G., Bissett, R. T., Pistorello, J., Toarmino, D., et al. (2004). Measuring experiential avoidance: A preliminary test of a working model. The Psychological Record, 54, 553-578.

Hayes, S. C., Wilson, K. G., Gifford, E. V., Follette, V. M., \& Strosahl, K. (1996). Experiential Avoidance and Behavioral Disorders: A Functional Dimensional Approach to Diagnosis and Treatment. Journal of Consulting and Clinical Psychology, 64(6), 1152-1168, available via: http://dx.doi: 10.1037/0022006X.64.6.1152

Luciano, M. C. \& Sonsoles, M. (2006). La terapia de aceptación y compromiso (ACT). Fundamentos, características y evidencia. Papeles del psicólogo, 27(2), 79-91.

Pankey, J. \& Hayes, S. C. (2003). Acceptance and Commitment Therapy for Psychosis. International Journal of Psychology and Psychological Therapy, 3 (2), 311-328.

Patrón, F. (2010). La Evitación Experiencial y su medición por medio del AAQ-II. Enseñanza e Investigación en Psicología, 15, 5-19.

Rodríguez, L. J. (2002). Síndrome de intestino irritable.- tratamiento psicológico desde el conductismo contextual. Ponencia en el 3er Congreso Virtual de Psiquiatría -Interpsiquis 2002.

Sidman, M. (1971). Reading and auditory-visual equivalences. Journal of Speech and Hearing Research, 14, 5-13.

Skinner, B. F. (1935). The generic nature of the Concepts of stimulus and response. Journal of General Psychology, 12, 40-65, available via: http://dx.doi.org/10.1080/00221309.19359920 087

Tafoya-Ramos, S., Pérez-Mitre, G., Ortega-Soto, H. \& Ortiz-León, S. (2006). Inventario de Ansiedad de Beck (BAI): validez y confiabilidad en estudiantes que solicitan atención psiquiátrica en la UNAM. Psiquis, 5(3), $82-87$.

Twohig, M., Shoenberger, D. \& Hayes, S. (2007). Preliminary investigation of Acceptance and Commitment Therapy as a treatment for marijuana Dependence in adults. Journal of applied behavior analysis, 40, 619632, available via: http://dx.doi: 10.1901/ jaba.2007.619-632 
Vargas, J. \& Aguilar, J. (2006). Psicopatología y Evitación Experiencial: AAQ-MEX, comparación de tres pequeñas muestras. Psicología y salud, 16, 249-252.

Velasco, J. A. \& Quiroga, E. (2001). Formulación y solución de un caso de abuso de alcohol en términos de aceptación y compromiso. Psicothema, 13(1), 50-56.

Wagner, F. A., González-Forteza, C., SánchezGarcía, s., García-Peña, C., \& Gallo, J. J. (2012). Enfocando la depresión como problema de salud en México. Salud Mental, 35, 3-11.

Wilson, K. G. \& Luciano, M. C. (2002) Terapia de Aceptación y Compromiso (ACT): un tratamiento conductual orientado a los valores. España: Pirámide.

Auto-referencias de autor: 1

Auto-referencias de la revista JBHSI: 0 